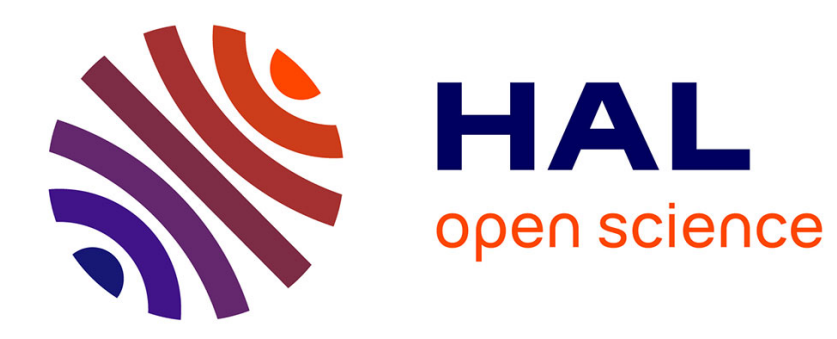

\title{
Luminosité en holographie de Fourier
}

\author{
G. Hepner, D. Poulbot
}

\section{To cite this version:}

G. Hepner, D. Poulbot. Luminosité en holographie de Fourier. Revue de Physique Appliquée, 1968, 3 (2), pp.157-168. 10.1051/rphysap:0196800302015700 . jpa-00242840

\section{HAL Id: jpa-00242840 https://hal.science/jpa-00242840}

Submitted on 1 Jan 1968

HAL is a multi-disciplinary open access archive for the deposit and dissemination of scientific research documents, whether they are published or not. The documents may come from teaching and research institutions in France or abroad, or from public or private research centers.
L'archive ouverte pluridisciplinaire HAL, est destinée au dépôt et à la diffusion de documents scientifiques de niveau recherche, publiés ou non, émanant des établissements d'enseignement et de recherche français ou étrangers, des laboratoires publics ou privés. 


\title{
LUMINOSITÉ EN HOLOGRAPHIE DE FOURIER
}

\author{
Par G. HEPNER et D. POULBOT, \\ Laboratoires de Recherches Générales de la Compagnie Française Thomson Houston-Hotchkiss Brandt.
}

(Reçu le 21 novembre 196\%.)

\begin{abstract}
Résumé. - Le but du présent article est de montrer quelle luminosité peut être atteinte en holographie de Fourier, aussi bien en réponse impulsionnelle (compte tenu de la résolution désirée) qu'en corrélation. Les résultats expérimentaux décrits ont été obtenus par holographie de Fourier à affichage symétrique, procédé linéaire, qui, de ce fait, conduit à une luminosité supérieure à performance égale.
\end{abstract}

Abstract. - Discussion of the luminosity in Fourier holography for a given resolution both in pulse response and in correlation. The experimental results were obtained in Fourier holography where the desired function is taken with its symmetric. This gives a linear process and the luminosity is found superior to classical holography for the same performances.

L'holographie de Fourier. Définition d'un hologramme de Fourier. - On sait que la propriété essentielle des calculateurs optiques cohérents est la simplicité avec laquelle ils permettent d'effectuer une transformation de Fourier à deux dimensions. En effet, la répartition d'amplitude dans le plan focal image d'un objectif est la transformée de Fourier de la répartition d'amplitude dans le plan focal objet $[1,2]$. Il suffit donc, pour faire cette opération, de

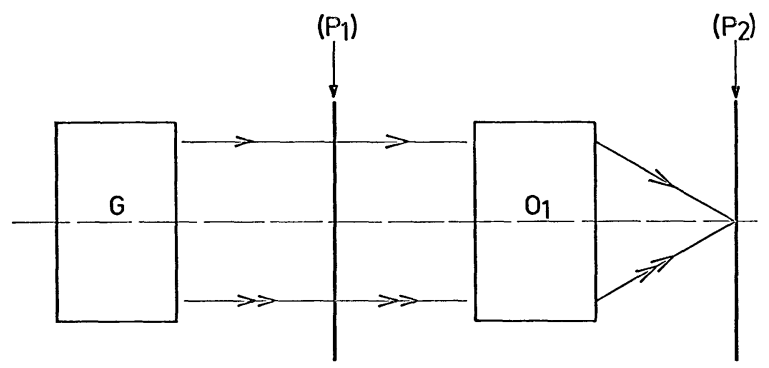

FIg. 1. - Propriété essentielle des calculateurs optiques cohérents. - G : générateur d'ondes planes monochromatiques; $\mathrm{P}_{1}$ : plan d'affichage; $\mathrm{O}_{1}$ : objectif formant en $\left(\mathrm{P}_{2}\right)$ la T.F. de $\left(\mathrm{P}_{1}\right) ; \mathrm{P}_{2}$ : plan de la transformée de Fourier (T.F.).

placer les signaux dans le plan $\left(\mathbf{P}_{\mathbf{1}}\right)$ de la figure 1 sous forme de transparence en amplitude $T_{1}\left(x_{1}, y_{1}\right)$ et d'éclairer ce plan en ondes planes monochromatiques pour obtenir en $\left(\mathrm{P}_{2}\right)$ la transformée de Fourier de $T_{1}\left(x_{1}, y_{1}\right)$ sous forme de répartition d'amplitude.
Gette transformée étant généralement complexe, il faut utiliser une technique holographique pour pouvoir l'enregistrer photographiquement sans perte d'informations. Le cliché ainsi obtenu constitue un hologramme de Fourier.

L'holographie de Fourier classique. - Ordinairement, les hologrammes de Fourier sont obtenus en ajoutant à la transformée de Fourier une onde plane oblique.

C'est le procédé mis en œuvre par le montage de la figure 2, dû à Van der Lugt [3]. Oń dispose en $\left(\mathbf{P}_{1}\right)$

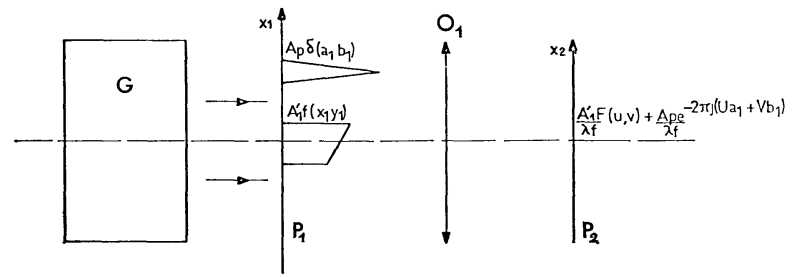

FIG. 2. - Holographie de Fourier ordinaire (enregistrement de l'hologramme). - G : générateur d'ondes planes monochromatiques; $\mathrm{P}_{1}$ : plan d'affichage ; $\mathrm{O}_{1}$ : objectif formant en $\mathrm{P}_{2}$ la transformée de Fourier de $\mathrm{P}_{1} ; \mathrm{P}_{2}$ : plan de la T.F. de $\mathrm{P}$ où est disposée la plaque photographique.

éclairé par une onde d'amplitude $\mathrm{A}_{1}^{\prime}$ une transparence $f\left(x_{1}, y_{1}\right)$ et une impulsion de Dirac décentrée, $\lambda f A_{\mathrm{p}} \delta\left(a_{1}, b_{1}\right)$. 
La répartition d'amplitude en $\left(\mathrm{P}_{2}\right)$ est alors :

$$
F^{\prime}(U, V)=\frac{A_{1}^{\prime}}{\lambda f} F(U, V)+A_{\mathrm{p}} \mathrm{e}^{-2 \pi j\left(U a_{1}+V b_{1}\right)}
$$

avec $\lambda=$ longueur d'onde de la raie utilisée $f=$ focale de $\mathrm{O}_{1}$

$F(U, V)=\mathscr{F}\left[f\left(x_{1}, y_{1}\right)\right]=$ transformée de Fourier

$$
\begin{aligned}
& \operatorname{de} f\left(x_{1}, y_{1}\right) \\
U= & x_{2} / \lambda f \\
V= & y_{2} / \lambda f .
\end{aligned}
$$

La plaque photographique est sensible à l'intensité :

$$
I=F^{\prime}(U, V) F^{\prime *}(U, V) \text {. }
$$

La courbe de réponse en amplitude du processus photographique quadratique utilisé (facteur de contraste $\Gamma=-2$ ) de la figure $4 \mathrm{a}$, conduit à une transparence en amplitude qui peut être donnée sous la forme d'un développement en série autour du point $\left(I_{0}=A_{\mathrm{p}}^{2}, t_{0}\right)$ :

$$
\begin{aligned}
t(U, V)=t_{0}+k_{1}\left(I-I_{0}\right)+ & k_{2}\left(I-I_{0}\right)^{2} \\
& +k_{3}\left(I-I_{0}\right)^{3}+\ldots
\end{aligned}
$$

où $t_{0}, k_{1}, k_{2}, k_{3}$ sont des constantes pour un temps de pose et un processus photographique donnés.

En supposant que l'excursion autour de $\left(I_{0}, t_{0}\right)$ est petite (l'influence des non-linéarités quand elle devient importante est traitée en annexe), on peut écrire :

$$
t(U, V)=t_{0}+k_{1}\left(I-I_{0}\right)
$$

soit, en tenant compte de (1) et (2) :

$$
\begin{aligned}
t(U, V)=t_{0}+k_{1} & {\left[\frac{A_{1}^{\prime}}{\lambda f} F(U, V) A_{\mathrm{p}} \mathrm{e}^{+2 \pi j\left(U a_{1}+V b_{1}\right)}\right.} \\
& +\frac{A_{1}^{\prime}}{\lambda f} F^{*}(U, V) A_{\mathrm{p}} \mathrm{e}^{-2 \pi j\left(U a_{1}+V b_{1}\right)} \\
& \left.+\left(\frac{A_{1}^{\prime}}{\lambda f}\right)^{2} F(U, V) F^{*}(U, V)\right]
\end{aligned}
$$

où il apparaît que la transformée de Fourier de $f\left(x_{1}, y_{1}\right)$ a bien été enregistrée autour d'une fréquence porteuse dont témoigne le facteur $\mathrm{e}^{+2 \pi j\left(U a_{1}+V b_{1}\right)}$.

L'holographie de Fourier à affichage symétrique. - L'arrangement utilisé est représenté par la figure 3. On affiche en $\left(\mathrm{P}_{1}\right)$ éclairé par une onde d'amplitude $A_{1}^{\prime}$ la fonction $f\left(x_{1}, y_{1}\right)$ translatée de $a_{2}$ et $b_{2}$,

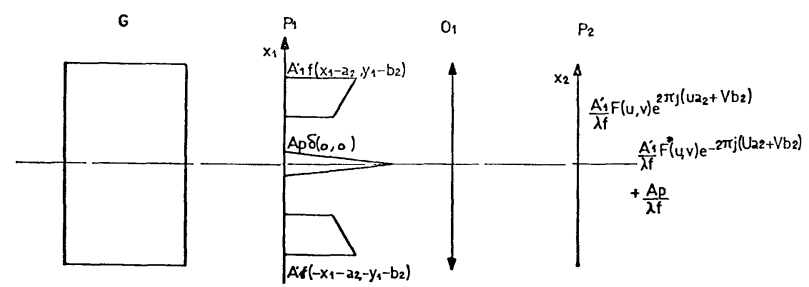

Fig. 3. - Holographie de Fourier symétrique (enregistrement de l'hologramme). - G : générateur d'ondes planes monochromatiques; $\mathrm{P}_{1}$ : plan d'affichage; $\mathrm{O}_{1}$ : objectif formant en $\mathrm{P}_{2}$ la T.F. de $\mathrm{P}_{1} ; \mathrm{P}_{2}$ : plan de la T.F. où est disposée la plaque photographique. soit $f\left(x_{1}-a_{2}, y_{1}-b_{2}\right)$, ainsi que le symétrique $f\left(-x_{1}-a_{2},-y_{1}-b_{2}\right)$, et une impulsion de Dirac centrée $\lambda f A_{\mathrm{p}} \delta(0,0)$.

D'où la répartition d'amplitude en $\left(\mathrm{P}_{2}\right)$ :

$$
\begin{aligned}
F^{\prime}(U, V)= & \frac{A_{1}^{\prime}}{\lambda f} F(U, V) \mathrm{e}^{+2 \pi j\left(U a_{\mathbf{z}}+V b_{\mathbf{z}}\right)} \\
& +\frac{A_{1}^{\prime}}{\lambda f} F^{*}(U, V) \mathrm{e}^{-2 \pi j\left(U a_{\mathbf{z}}+V b_{\mathbf{z}}\right)}+A_{\mathrm{p}} .
\end{aligned}
$$

Les deux premiers termes de (5) étant conjugués complexes, leur somme est réelle, donc, si $A_{\mathrm{p}}$ est suffisante, la somme des trois termes sera réelle et positive. $F^{\prime}(U, V)$ peut alors être enregistrée photographiquement sans perte d'information.

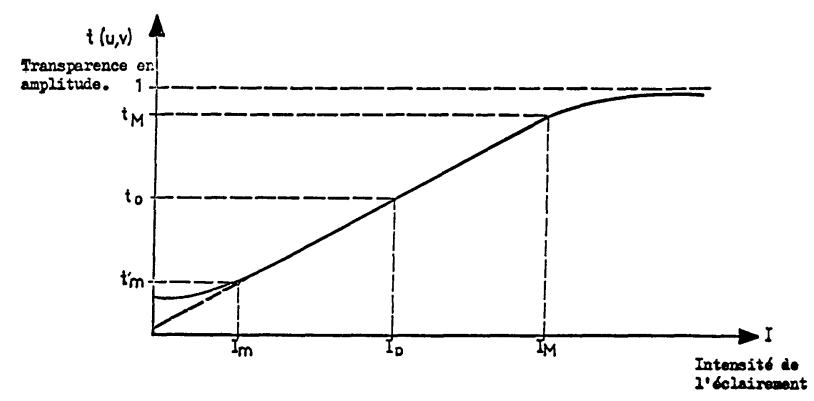

Fig. 4 a. - Courbe caractéristique du processus photographique (à temps de pose constant) pour l'holographie de Fourier ordinaire.

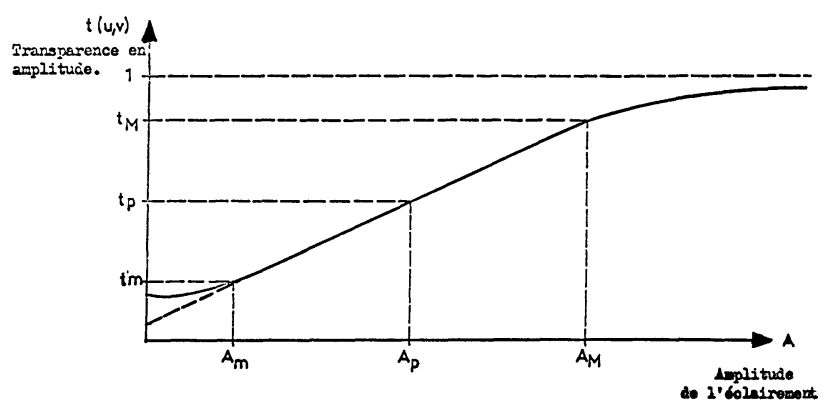

FIG. $4 \mathrm{~b}$. - Courbe caractéristique du processus photographique (à temps de pose constant) pour l'holographie symétrique.

Un processus photographique linéaire (facteur de contraste $\Gamma=-1$ ) dont la courbe de réponse en amplitude serait identique à celle de la figure $4 \mathrm{~b}$ fournit un cliché dont la transparence en amplitude peut être donnée sous la forme d'un développement en série autour du point $\left(A_{\mathbf{0}}, t_{\mathbf{0}}\right)$ :

$$
\begin{aligned}
t(U, V)=t_{0}+k_{1}\left(A-A_{0}\right) & +k_{2}\left(A-A_{0}\right)^{2} \\
& +k_{3}\left(A-A_{0}\right)^{3}+\ldots
\end{aligned}
$$

où $A_{0}=A_{\mathrm{p}}, A=F^{\prime}(U, V)$ et $t_{0}, k_{1}, k_{2}, k_{3}$ sont des constantes pour un temps de pose et un processus photographique donnés. 
Ici aussi, pour une excursion faible autour de $\left(A_{0}, t_{0}\right)$, les termes non linéaires peuvent être négligés (leur influence est traitée en annexe pour des excursions plus importantes) et il vient :

$$
t(U, V)=t_{0}+k_{1}\left(A-A_{0}\right)
$$

soit, en tenant compte de (1) :

$$
\begin{aligned}
t(U, V)=t_{0}+k_{1}[ & \frac{A_{1}^{\prime}}{\lambda f} F(U, V) \mathrm{e}^{+2 \pi j\left(U a_{\mathbf{2}}+V b_{\mathbf{z}}\right)} \\
& \left.+\frac{A_{1}^{\prime}}{\lambda f} F^{*}(U, V) \mathrm{e}^{-2 \pi j\left(U a_{\mathbf{z}}+V b_{\mathbf{z}}\right)}\right]
\end{aligned}
$$

où il apparaît que la transformée de Fourier de $f\left(x_{1}, y_{1}\right)$ a bien été enregistrée autour d'une fréquence porteuse dont témoigne le facteur $\mathrm{e}^{+2 \pi j\left(U a_{2}+V b_{2}\right)}$.

Montage expérimental utilísé. - L'enregistrement des hologrammes de Fourier par la méthode de l'affichage symétrique a été réalisé au moyen du montage schématisé par la figure 5 .

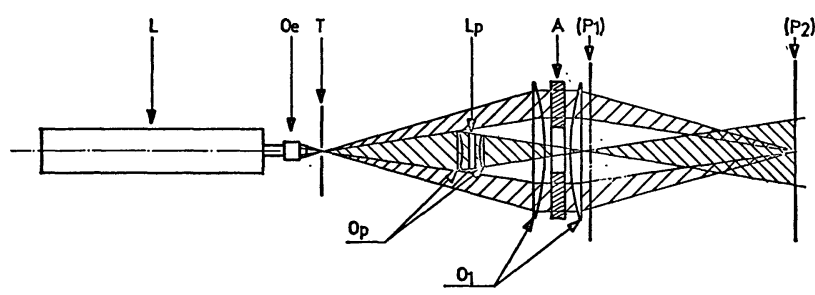

FIG. 5. - Montage expérimental utilisé pour enregistrer les hologrammes. - $\mathrm{L}$ : laser; Oe : objectif ; $\mathrm{T}$ : trou de filtrage ; Op : objectif fournissant en $\left(\mathrm{P}_{1}\right)$ l'impulsion de Dirac $A_{p} \delta(0,0) ; O_{1}$ : objectif formant en $\left(P_{2}\right)$ la transformée de Fourier de $\left(\mathrm{P}_{1}\right) ; \mathrm{P}_{1}$ : plan d'affichage ; $\mathrm{P}_{2}$ : plan de la transformée de Fourier; $\mathrm{A}$ : atténuateur.

Le faisceau issu du laser $\mathrm{L}$, réglé en monomode spatial, est focalisé sur un trou de filtrage de $10 \mu \mathrm{m}$ de diamètre grâce auquel le faisceau est " nettoyé ".

La disposition par rapport au doublet $\mathrm{O}_{1}$ du plan d'affichage, très classique [1], fournit en $\left(\mathrm{P}_{2}\right)$ la transformée de Fourier de $\left(\mathrm{P}_{\mathbf{1}}\right)$ à un coefficient de phase sphérique près, sans importance pour un enregistrement photographique.

Une partie du faisceau est interceptée par le doublet Oe pour former en $\left(\mathrm{P}_{\mathbf{1}}\right)$ l'impulsion $\lambda f A_{\mathrm{p}} \delta(0,0)$. La lame à faces parallèles $L p$ permet d'ajuster la phase de cette impulsion à la phase du faisceau principal qui vient éclairer l'affichage symétrique de $\left(\mathrm{P}_{1}\right)$. A est un atténuateur, percé d'un trou, dont le rôle consiste à assurer en $\mathrm{P}_{2}$ une valeur de l'amplitude du fond cohérent au moins égale au maximum de celle de la transformée de Fourier des deux fonctions affichées symétriquement.

Exemple d'obtention d'un hologramme de Fourier par la méthode de l'affichage symétrique. - La figure 6 a montre la transformée de Fourier bien connue d'une ouverture rectangulaire.
$6 \mathrm{~b}$ donne l'allure de la transformée de Fourier obtenue en affichant une seconde ouverture rectangulaire symétriquement par rapport à l'axe optique.

6 c représente la même transformée de Fourier additionnée d'une onde de référence introduite en affichant une fonction $\delta$ sur l'axe optique. On note que l'effet de redressement dû à la détection quadratique de la plaque photographique est supprimé et l'interfrange apparaît double.

Luminosité des images restituées. - Galcul thÉoRIQUE. - Quand un hologramme obtenu par la méthode de l'affichage symétrique est disposé dans le plan $\left(\mathbf{P}_{\mathbf{1}}\right)$ de la figure 2 , éclairé par une onde d'amplitude $A_{1}$, il se forme en $\left(\mathrm{P}_{2}\right)$ la transformée de Fourier de $A_{1} t(U, V)$, soit, d'après (7) :

$$
\begin{array}{r}
A_{1} t(U, V)=A_{1} t_{0}+A_{1} k_{1} \frac{A_{1}^{\prime}}{\lambda f}\left[F(U, V) \mathrm{e}^{+2 \pi j\left(U a_{2}+V b_{z}\right)}\right. \\
\left.+F^{*}(U, V) \mathrm{e}^{-2 \pi j\left(U a_{2}+V b_{2}\right)}\right]
\end{array}
$$

c'est-à-dire une image réelle des fonctions affichées en $\left(\mathbf{P}_{\mathbf{1}}\right)$ lors de l'enregistrement de l'hologramme.

Notamment, le terme redonnant l'image de $f\left(x_{1}-a_{2}, y_{1}-b_{2}\right)$ est :

$$
k_{1} \frac{A_{1} A_{1}^{\prime}}{\lambda f} F(U, V) \mathrm{e}^{+2 \pi j\left(U a_{2}+V b_{2}\right)} .
$$

Il contient une puissance totale $P_{\mathrm{i}}$ dont l'expression est :

$$
\begin{aligned}
P_{\mathrm{i}}=\left(k_{1} \frac{A_{1} A_{1}^{\prime}}{\lambda f}\right)^{2} & \\
& \quad \iint\left|F(U, V) \mathrm{e}^{-2 \pi j\left(U a_{2}+V b_{2}\right)}\right|^{2} \lambda f \mathrm{~d} U . \lambda f \mathrm{~d} V
\end{aligned}
$$

comme :

$$
\iint|F(U, V)|^{2} \mathrm{~d} U \mathrm{~d} V=\iint\left|f\left(x_{1}, y_{1}\right)\right|^{2} \mathrm{~d} x_{1} \mathrm{~d} y_{1}
$$

d'après le théorème de Parseval. Cette puissance est la même que celle contenue dans l'image restituée, soit :

$$
P_{\mathrm{i}}=\left(k_{1} A_{1} A_{1}^{\prime}\right)^{2} \iint\left|f\left(x_{1}, y_{1}\right)\right|^{2} \mathrm{~d} x_{1} \mathrm{~d} y_{1} .
$$

D'après la figure $4 \mathrm{~b}$, il vient :

$$
k_{1}=\frac{t_{\mathrm{M}}-t_{\mathrm{m}}}{A_{\mathrm{M}}-A_{\mathrm{m}}}=\frac{\Delta t_{2}}{A_{\mathrm{M}}-A_{\mathrm{m}}}
$$

en posant :

$$
\Delta t_{2}=t_{\mathrm{M}}-t_{\mathrm{m}}
$$

Si $F_{M}$ est la valeur maximum de la transformée de Fourier de $f\left(x_{1}, y_{1}\right)$ seule, on peut écrire :

$$
\begin{aligned}
A_{\mathrm{M}}-A_{\mathrm{m}}=\left[\frac{A_{\mathrm{p}}}{\lambda f}+\right. & \left.2 \frac{A_{1}^{\prime}}{\lambda f} F_{\mathrm{M}}\right] \\
& -\left[\frac{A_{\mathrm{p}}}{\lambda f}-2 \frac{A_{1}^{\prime}}{\lambda f} F_{\mathrm{M}}\right]=4 \frac{A_{1}^{\prime}}{\lambda f} F_{\mathbf{M}} \\
\text { d'où : } & k_{1}=\frac{\Delta t_{2} \lambda f}{4 A_{1}^{\prime} F_{\mathrm{M}}} .
\end{aligned}
$$




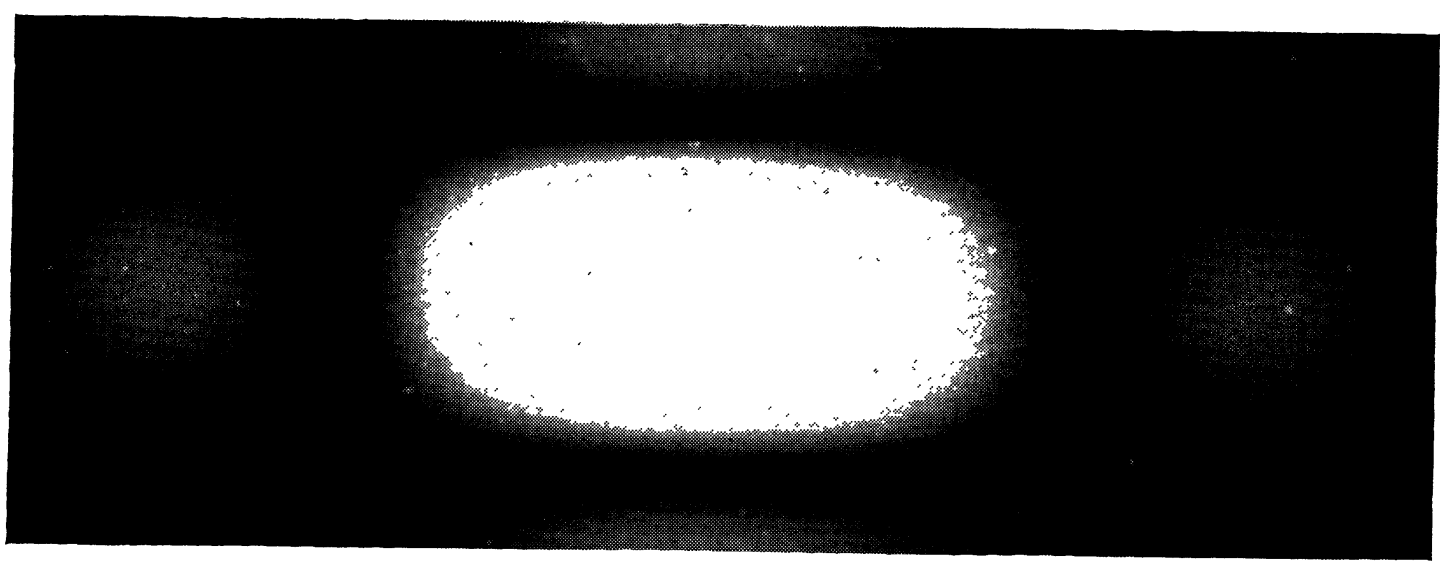

a) Une fente seule.

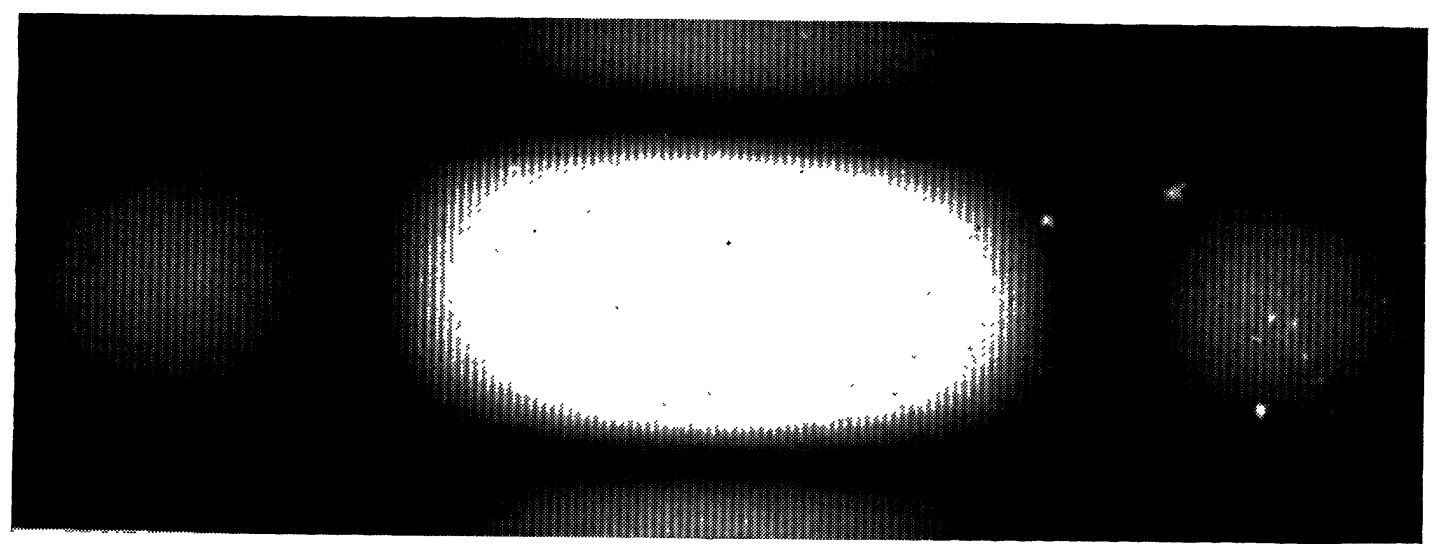

b) Une fente avec symétrique.

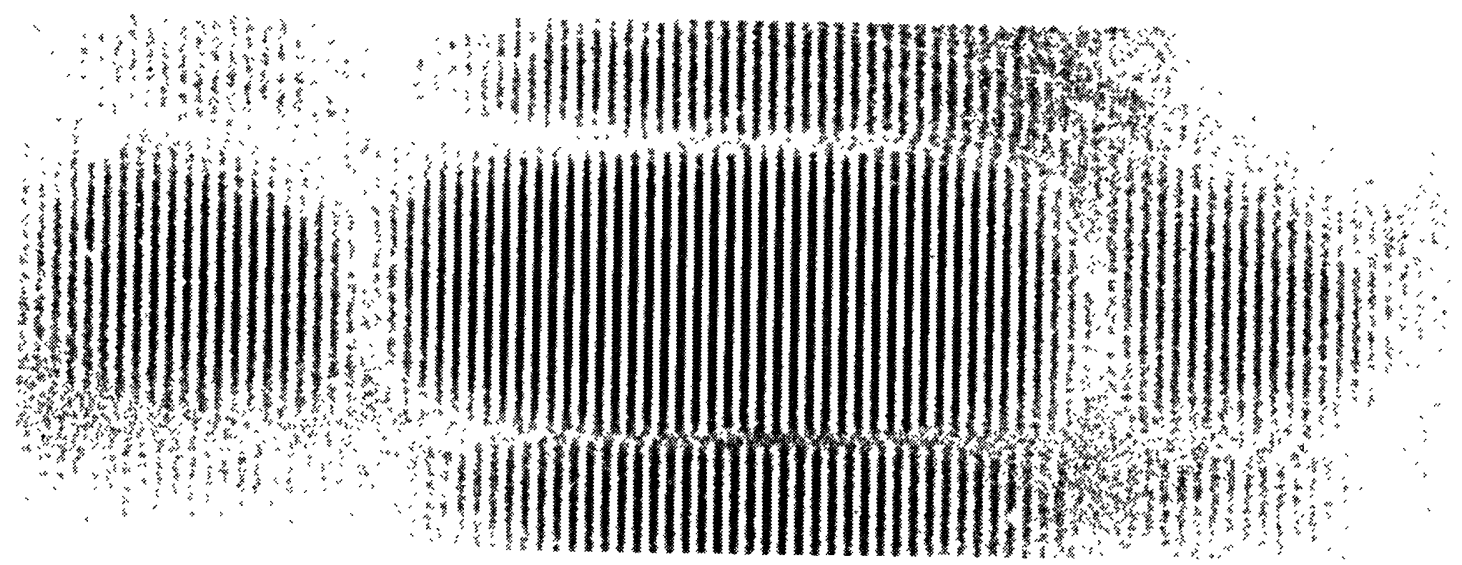

c) Une fente avec symétrique et polarisation.

FIG. 6. - Transformée de Fourier d'une fente. 
Reportons cette valeur dans l'expression de $P_{\mathrm{i}}$ :

$$
\begin{aligned}
P_{\mathrm{i}} & =\left(\frac{\Delta t_{2} \lambda f}{4 A_{1}^{\prime} F_{\mathrm{M}}} A_{1} A_{1}^{\prime}\right)^{2} \iint\left|f\left(x_{1}, y_{1}\right)\right|^{2} \mathrm{~d} x_{1} \mathrm{~d} y_{1} \\
& =\left(\frac{\Delta t_{2} \lambda f A_{1}}{4 F_{\mathrm{M}}}\right)^{2} \iint\left|f\left(x_{1}, y_{1}\right)\right|^{2} \mathrm{~d} x_{1} \mathrm{~d} y_{1} .
\end{aligned}
$$

Dans la plupart des cas, $f\left(x_{1}, y_{1}\right)$ est réelle et positive (cliché photographique), et dans ce cas :

$$
F_{\mathrm{M}}=\iint f\left(x_{1}, y_{1}\right) \mathrm{d} x_{1} \mathrm{~d} y_{1}
$$

d'où :

$$
P_{\mathrm{i}}=\left(\frac{\Delta t_{2} \lambda f A_{1}}{4}\right)^{2} \frac{\iint\left|f\left(x_{1}, y_{1}\right)\right|^{2} \mathrm{~d} x_{1} \mathrm{~d} y_{1}}{\left(\iint f\left(x_{1}, y_{1}\right) \mathrm{d} x_{1} \mathrm{~d} y_{1}\right)^{2}}
$$

En supposant la puissance $P$, émise par le laser, uniformément répartie dans un cercle de diamètre $D$ au niveau de $\left(\mathrm{P}_{1}\right)$, on a :

$$
P=A_{1}^{2} \pi D^{2} / 4
$$

Par ailleurs, la résolution linéaire dans le plan $\left(\mathrm{P}_{2}\right)$ est donnée par :

$$
d=1,22 \lambda f \mid D
$$

En éliminant $D$ entre ces deux relations et en reportant la valeur de $A_{1}^{2}$ ainsi obtenue en (9), on trouve :

$$
\begin{aligned}
& P_{\mathrm{i}}=\left(\frac{\Delta t_{2} \lambda f}{4}\right)^{2} \frac{4 P d^{2}}{\pi(1,22 \lambda f)^{2}} \frac{\iint\left|f\left(x_{1}, y_{1}\right)\right|^{2} \mathrm{~d} x_{1} \mathrm{~d} y_{1}}{\left(\iint f\left(x_{1}, y_{1}\right) \mathrm{d} x_{1} \mathrm{~d} y_{1}\right)^{2}} \\
& P_{\mathrm{i}}=\frac{P}{\pi}\left(\frac{\Delta t_{2} d}{1,22}\right)^{2} \frac{\iint\left|f\left(x_{1}, y_{1}\right)\right|^{2} \mathrm{~d} x_{1} \mathrm{~d} y_{1}}{\left(\iint f\left(x_{1}, y_{1}\right) \mathrm{d} x_{1} \mathrm{~d} y_{1}\right)^{2}} .
\end{aligned}
$$

Dans la plupart des applications pratiques, $f\left(x_{1}, y_{1}\right)$ est une fonction binaire à deux niveaux correspondant à des surfaces transparentes ou opaques. Dans ce cas, si $S$ est la surface totale transparente, on a :

$$
S=\iint\left|f\left(x_{1}, y_{1}\right)\right|^{2} \mathrm{~d} x_{1} \mathrm{~d} y_{1}=\iint f\left(x_{1}, y_{1}\right) \mathrm{d} x_{1} \mathrm{~d} y_{1}
$$

et la formule (10) s'écrit :

$$
P_{\mathrm{i}}=\frac{P}{\pi(1,22)^{2}}\left(\Delta t_{2}\right)^{2} \frac{d^{2}}{S}
$$

ou encore :

$$
P_{\mathrm{i}}=\frac{P}{\pi(1,22)^{2}}\left(\Delta t_{2}\right)^{2} \frac{1}{N} .
$$

Donc, pour une puissance laser donnée et un nombre de points images indépendants déterminé, $N=d^{2} / S$, la puissance totale répartie sur une image obtenue à partir d'un hologramme de Fourier ne dépend que de la dynamique utilisable $\Delta t_{2}$ de la courbe de réponse en amplitude du processus photographique utilisé pour obtenir l'hologramme.
Le même calcul mené en holographie ordinaire aboutit au même résultat mais avec une dynamique $\Delta t_{1}$ du processus photographique se rapportant à une courbe de réponse en intensité comme celle de la figure $4 \mathrm{a}$.

Vérification eXPÉRIMENTALE. - Application à la luminosité d'une image holographique constituée par une fonction longiligne du type caractère d'imprimerie.

Pour ce genre de fonctions, la largeur du trait doit être de l'ordre de $d$. En posant $l=$ longueur curviligne de la lettre ou de l'élément de lettre, la relation (11) devient :

$$
P_{\mathrm{i}}=\frac{P}{\pi(1,22)^{2}}\left(\Delta t_{2}\right)^{2} \frac{d}{l}
$$

tandis que l'intensité relevée sur l'image a pour expression :

$$
\begin{aligned}
& I=\frac{P_{\mathrm{i}}}{l d} . \\
& I=\frac{P}{\pi(1,22)^{2}}\left(\Delta t_{2}\right)^{2} \frac{1}{l^{2}} .
\end{aligned}
$$

Cette formule a été vérifiée expérimentalement en comparant au photomultiplicateur l'intensité obtenue à partir des hologrammes de Fourier de deux objets constitués l'un par un trait de $0,4 \times 1 \mathrm{~mm}$, l'autre par une série de trois traits identiques.

Sur la première image, la tension relevée au photomultiplicateur $v_{1}$ est de $30 \mathrm{mV}$ contre $v_{2}=4,5 \mathrm{mV}$ sur la seconde image. On vérifie alors que le rapport expérimental :

$$
v_{1} / v_{2}=I_{1} / I_{2}=30 / 4,5=7
$$

est sensiblement égal au rapport théorique :

$$
I_{1} / I_{2}=\left(3 l_{1} / l_{1}\right)^{2}=9 \text {. }
$$

Cet effet est également visible sur le sigle de la figure 7 , obtenu en photographiant successivement avec un même temps de pose les images hologra-

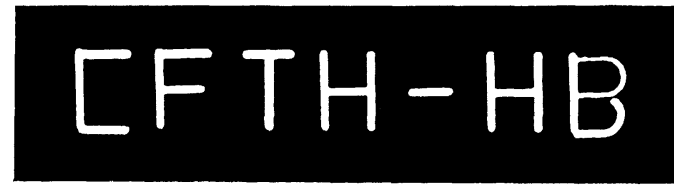

FIG. 7. - Réponse impulsionnelle des hologrammes de Fourier réalisés par la méthode de l'affichage symétrique.

phiques d'un certain nombre d'éléments de lettres. L'élément constituant la boucle supérieure du B présentant une longueur à peu près double de celle des autres éléments, il aurait fallu poser quatre fois plus longtemps pour avoir la même luminosité sur le cliché. 
Application de l'holographie de Fourier à la reconnaissance des formes. - Filtrage ADAPTÉ OPTIQUE ET REGONNAISSANGE DES FORMES. - Un important domaine d'application du calcul optique cohérent se rapporte à la reconnaissance de formes par filtrage adapté [3].

Le bloc diagramme d'un montage à filtrage adapté optique est donné par la figure 8 . On retrouve les

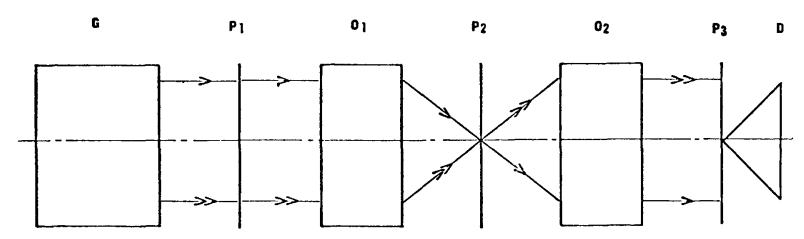

FIG. 8. - Schéma simplifié d'un calculateur optique cohérent. - G : générateur d'ondes planes monochromatiques ; $\mathrm{P}_{1}$ : plan primaire objet ou plan d'affichage : $\mathrm{O}_{1}$ : objectif formant en $\mathrm{P}_{2}$ la T.F. de $\mathrm{P}_{1} ; \mathrm{P}_{2}$ : plan de la transformée de Fourier; $\mathrm{O}_{2}$ : objectif formant en $\mathrm{P}_{3}$ la T.F. de $\mathrm{P}_{2} ; \mathrm{P}_{3}$ : plan de sortie ; $\mathrm{D}$ : détecteur photoélectrique.

mêmes éléments que sur la figure 1 , suivis par une seconde optique $\mathrm{O}_{2}$ qui reforme en $\left(\mathrm{P}_{3}\right)$ la transformée de Fourier de $\left(\mathrm{P}_{2}\right)$. Donc, si une transparence variable $t_{2}\left(x_{2}, y_{2}\right)$ est disposée en $\left(\mathrm{P}_{2}\right)$, la répartition d'amplitude obtenue en $\left(\mathrm{P}_{3}\right)$ n'est autre que le produit de convolution de la fonction affichée en $\left(\mathrm{P}_{1}\right), f\left(x_{1}, y_{1}\right)$, par la transformée de Fourier de $t_{2}\left(x_{2}, y_{2}\right)$, si :

et :

$$
F(U, V)=\mathscr{F}\left[f\left(x_{1}, y_{1}\right)\right]
$$

$$
t_{2}\left(x_{2}, y_{2}\right)=F^{*}(U, V) .
$$

Le résultat obtenu en $\left(\mathrm{P}_{3}\right)$ sera la fonction d'autocorrélation de $f\left(x_{1}, y_{1}\right)$. L'existence du maximum de cette fonction permet de conclure que $f\left(x_{1}, y_{1}\right)$ est bien présente en $\left(\mathbf{P}_{\mathbf{1}}\right)$, tandis que sa position en $\left(\mathrm{P}_{\mathbf{3}}\right)$ correspond à celle de $f\left(x_{1}, y_{1}\right)$ en $\left(\mathbf{P}_{1}\right)$.

Un tel montage est donc susceptible de discerner si un signal est affiché en $\left(\mathbf{P}_{\mathbf{1}}\right)$ et en quel endroit de $\left(\mathrm{P}_{\mathbf{1}}\right)$ il se trouve.

Ce signal peut être une fonction d'une seule variable comme un signal radar noyé dans un bruit de fond [4] ou une fonction de deux variables comme un mot au milieu d'une page de texte [5]. La principale difficulté à surmonter pour la mise en ouvre de cette technique réside dans l'élaboration du filtre adapté dont la fonction de transfert est :

$\frac{F^{*}(U, V)}{N^{2}(U, V)}$

Conjugué complexe

de la transformée de Fourier du signal

Densité spectrale énergétique du bruit

qui se réduit à $F^{*}(U, V)$ quand le bruit est blanc dans la bande passante du signal.
En général, cette fonction est complexe, donc il est impossible de l'enregistrer directement sous forme de cliché photographique à moins d'utiliser les techniques holographiques.

Application de L'holographie de Fourier clasSIQUE A LA REGONNAISSANGE DES FORMES. — Van der Lugt a proposé le premier une solution élégante qui consiste à utiliser comme filtre adapté un hologramme de Fourier classique [3].

Le schéma de la figure 9 montre le fonctionnement

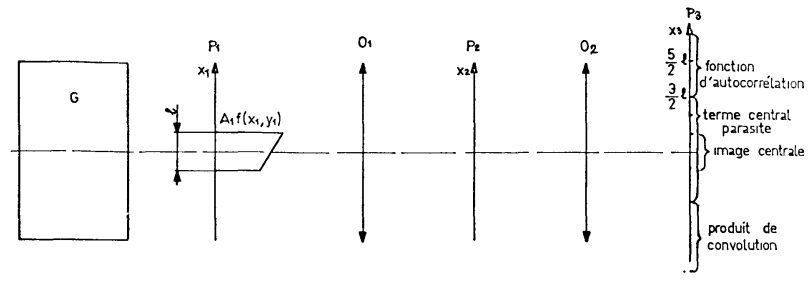

FIG. 9. - Holographie de Fourier ordinaire (exploitation de l'hologramme en corrélation). - G : générateur d'ondes planes monochromatiques; $\mathrm{P}_{1}$ : plan d'affichage; $\mathrm{O}_{1}$ : objectif formant en $\mathrm{P}_{2}$ la T.F. de $\mathrm{P}_{1}$; $\mathrm{P}_{2}$ : plan de la T.F. où est inséré l'hologramme obtenu par le montage de la figure $2 ; \mathrm{O}_{2}$ : objectif formant en $\mathrm{P}_{3}$ la T.F. de $\mathrm{P}_{2}$.

d'un tel montage de filtrage adapté. La fonction à détecter $f\left(x_{1}, y_{1}\right)$ est affichée en $\left(\mathbf{P}_{\mathbf{1}}\right)$, éclairée par une onde d'amplitude $A_{1}$.

Il se forme donc, en $\left(\mathrm{P}_{2}\right), \frac{A_{1}}{\lambda f} F(U, V)$, et sitôt après $\left(\mathrm{P}_{2}\right)$, où est disposée la transparence $t_{2}\left(x_{2}, y_{2}\right)$, la répartition d'amplitude est devenue :

$$
A_{2}\left(x_{2}, y_{2}\right)=\frac{A_{1}}{\lambda f} F(U, V) t_{2}\left(x_{2}, y_{2}\right)
$$

soit, d'après (4) :

$$
\begin{aligned}
& A_{2}\left(x_{2}, y_{2}\right)=\frac{A_{1}}{\lambda f} F(U, V) \\
& \qquad \begin{aligned}
\left\{t_{0}\right. & +\frac{k_{1}}{\lambda f}\left[A_{\mathrm{p}} A_{1}^{\prime} F(U, V) \mathrm{e}^{+2 \pi j\left(U a_{1}+V b_{1}\right)}\right. \\
& +A_{\mathrm{p}} A_{1}^{\prime} F^{*}(U, V) \mathrm{e}^{-2 \pi j\left(U a_{1}+V b_{1}\right)} \\
& \left.\left.+\frac{A_{1}^{\prime 2}}{\lambda f} F(U, V) F^{*}(U, V)\right]\right\}
\end{aligned}
\end{aligned}
$$

La transformée de Fourier obtenue en $\left(\mathrm{P}_{3}\right)$ comprendra donc quatre termes dont celui correspondant à :

$$
\frac{A_{1}}{\lambda f} F(U, V) \frac{A_{1}^{\prime} A_{\mathrm{p}}}{\lambda f} F^{*}(U, V) \mathrm{e}^{2 \pi j\left(U a_{1}+V b_{1}\right)}
$$

qui est la fonction d'autocorrélation recherchée, translatée de $\sqrt{a_{1}^{2}+b_{1}^{2}}$, donc séparée spatialement des trois autres termes si cette translation est au moins égale à deux fois et demie la longueur de la fonction. 
Application de L'holographie de Fourier syméTRIQUE A LA REGONNAISSANGE DE FORMES. - Le montage de la figure 10 est identique à celui de la figure 9,

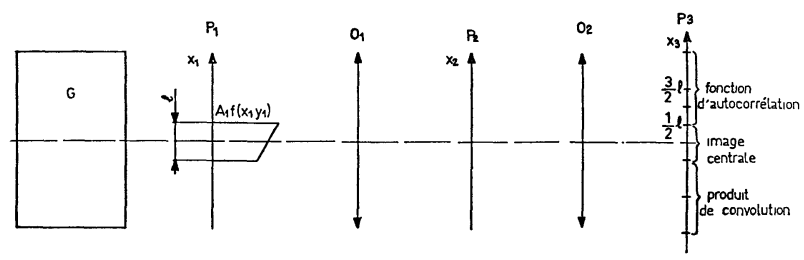

FIG. 10. - Holographie de Fourier symétrique (exploitation de l'hologramme en corrélation). - G : générateur d'ondes planes monochromatiques; $\mathrm{P}_{\mathbf{1}}$ : plan d'affichage; $\mathrm{O}_{1}$ : objectif formant en $\mathrm{P}_{2}$ la T.F. de $\mathrm{P}_{1}$; $\mathrm{P}_{2}$ : plan de la T.F. où est inséré l'hologramme obtenu par le montage de la figure $3 ; \mathrm{O}_{2}$ : objectif formant en $\mathrm{P}_{3}$ la T.F. de $\mathrm{P}_{2}$.

mais la transparence disposée en $\left(\mathrm{P}_{2}\right)$ est un hologramme de Fourier obtenu avec un affichage symétrique. Dans ce cas, la répartition d'amplitude sitôt après $\left(\mathrm{P}_{2}\right)$ est, d'après $(7)$ :

$$
\begin{aligned}
A_{2}\left(x_{2}, y_{2}\right)=\frac{A_{1}}{\lambda f} F(U, V) & \\
& \left\{t_{0}+k_{1} \frac{A_{1}^{\prime}}{\lambda f}\left[F(U, V) \mathrm{e}^{+2 \pi j\left(U a_{2}+V b_{2}\right)}\right.\right. \\
& \left.\left.+F^{*}(U, V) \mathrm{e}^{-2 \pi j\left(U a_{2}+V b_{2}\right)}\right]\right\} .
\end{aligned}
$$

Cette fois, la transformée de Fourier obtenue en $\left(\mathrm{P}_{3}\right)$ ne comporte que trois termes dont celui correspondant à :

$$
\frac{A_{1} A_{1}^{\prime}}{(\lambda f)} F(U, V) F^{*}(U, V) \mathrm{e}^{-2 \pi j\left(U a_{2}+V b_{2}\right)}
$$

qui est bien la fonction d'autocorrélation recherchée translatée de $\sqrt{a_{2}^{2}+b_{2}^{2}}$, donc séparée spatialement des deux autres termes pourvu que la translation soit au moins égale à une fois et demie la longueur de la fonction $f\left(x_{1}, y_{1}\right)$.

Montage EXPÉRIMENTAL UTILISÉ EN REGONNAISSANGE DE FORMES. - Le montage expérimental de corrélation représenté par la figure 11 est identique à celui

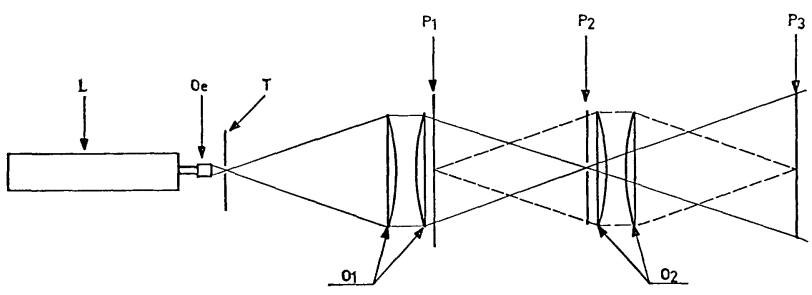

FrG. 11. - Montage expérimental utilisé en corrélation. - L : laser; Oe : objectif; $\mathrm{T}$ : trou de filtrage ; $\mathrm{O}_{1}$ : objectif formant en $\mathrm{P}_{2}$ la transformée de Fourier de $\mathrm{P}_{1} ; \mathrm{P}_{1}$ : plan d'affichage; $\mathrm{P}_{2}$ : plan de filtrage ; $\mathrm{O}_{2}$ : objectif formant en $\mathrm{P}_{3}$ la transformée de Fourier de $\mathrm{P}_{2} ; \mathrm{P}_{3}:$ plan de corrélation. de la figure 5 prolongé par un second objectif $\mathrm{O}_{2}$ et privé de $\mathrm{O}_{\mathrm{p}}$ et $\mathrm{A}$.

Le signal est affiché en $\left(P_{1}\right)$ seul ou mélangé à un bruit calibré et son filtre adapté disposé en $\left(\mathrm{P}_{2}\right)$.

La transformée de Fourier de la fonction d'affichage se forme en $\left(\mathrm{P}_{2}\right)$, affectée d'un coefficient de phase sphérique qui intervient sur la répartition d'amplitude $A_{2}\left(x_{2}, y_{2}\right)$ obtenue sitôt après $\left(\mathrm{P}_{2}\right)$. La première lentille de $\mathrm{O}_{2}$ annule ce coefficient de phase, tandis que la seconde fournit en $\left(\mathbf{P}_{\mathbf{3}}\right)$ la transformée de Fourier de $A_{2}\left(x_{2}, y_{2}\right)$ à un coefficient de phase sphérique près sans importance pour un enregistrement photographique ou une analyse au photomultiplicateur.

Luminosité du maximum de corrélation. - GalGul THÉORIQUe GÉNÉRAL. - Quand le filtre de $\left(\mathrm{P}_{\mathbf{2}}\right)$ est un hologramme de Fourier obtenu par la méthode de l'affichage symétrique, la répartition d'amplitude sitôt après $\left(\mathrm{P}_{2}\right)$ est, d'après (7) :

$$
\begin{aligned}
& A_{2}\left(x_{2}, y_{2}\right)=\frac{A_{1}}{\lambda f} F(U, V) \\
& \qquad t_{0}+k_{1} \frac{A_{1}^{\prime}}{\lambda f}\left[F(U, V) \mathrm{e}^{+2 \pi j\left(U a_{2}+V b_{2}\right)}\right. \\
&\left.\left.\quad+F^{*}(U, V) \mathrm{e}^{-2 \pi j\left(U a_{2}+V b_{2}\right)}\right]\right\} .
\end{aligned}
$$

D'où l'amplitude de la fonction de corrélation dans le plan $\left(\mathrm{P}_{3}\right)$ :

$$
\begin{aligned}
\Phi_{f f}\left(x_{3}, y_{3}\right)= & \lambda f \frac{A_{1} A_{1}^{\prime}}{(\lambda f)^{2}} k_{1} \\
& \iint_{\left(\mathrm{P}_{1}\right)} f\left(x_{1}, y_{1}\right) f\left(x_{1}-x_{3}, y_{1}-y_{3}\right) \mathrm{d} x_{1} \mathrm{~d} y_{1} .
\end{aligned}
$$

et en particulier l'amplitude de son maximum :

$$
\Phi_{f f}(0,0)=\frac{A_{1} A_{1}^{\prime}}{\lambda f} k_{1} \iint_{\left(\mathbf{P}_{1}\right)} f\left(x_{1}, y_{1}\right) f\left(x_{1}, y_{1}\right) \mathrm{d} x_{1} \mathrm{~d} y_{1}
$$

soit, en remplaçant $k_{1}$ par sa valeur de (8) :

$$
\Phi_{f f}(0,0)=\frac{A_{1} \Delta t_{2}}{4 F_{\mathrm{M}}} \iint_{\left(\mathrm{P}_{1}\right)} f\left(x_{1}, y_{1}\right) f\left(x_{1}, y_{1}\right) \mathrm{d} x_{1} \mathrm{~d} y_{1} .
$$

L'intensité du maximum de corrélation sera donc : $\left[\Phi_{f f}(0,0)\right]^{2}$

$$
=\left(\frac{A_{1} \Delta t_{2}}{4 F_{\mathrm{M}}}\right)^{2}\left[\iint_{\left(\mathrm{P}_{1}\right)} f\left(x_{1}, y_{1}\right) f\left(x_{1}, y_{1}\right) \mathrm{d} x_{1} \mathrm{~d} y_{1}\right]^{2} .
$$

Dans la plupart des cas, quand $f\left(x_{1}, y_{1}\right)$ est réelle et positive, on a :

$$
F_{\mathrm{M}}=\iint f\left(x_{1}, y_{1}\right) \mathrm{d} x_{1} \mathrm{~d} y_{1}
$$

d'où :

$$
\begin{aligned}
& {\left[\Phi_{f f}(0,0)\right]^{2}} \\
& \quad=\left(\frac{A_{1} \Delta t_{2}}{4}\right)^{2}\left[\frac{\iint f\left(x_{1}, y_{1}\right) f\left(x_{1}, y_{1}\right) \mathrm{d} x_{1} \mathrm{~d} y_{1}}{\iint f\left(x_{1}, y_{1}\right) \mathrm{d} x_{1} \mathrm{~d} y_{1}}\right]^{2} .
\end{aligned}
$$


Si de plus $f\left(x_{1}, y_{1}\right)$ est une fonction transparente ou opaque telle que la surface totale transparente soit $S$, on a :

$$
\iint f\left(x_{1}, y_{1}\right)^{2} \mathrm{~d} x_{1} \mathrm{~d} y_{1}=\iint f\left(x_{1}, y_{1}\right) \mathrm{d} x_{1} \mathrm{~d} y_{1}=S
$$

soit :

$$
\left[\Phi_{f f}(0,0)\right]^{2}=\left(\frac{A_{1} \Delta t_{2}}{4}\right)^{2} .
$$

Si la surface à éclairer est sensiblement double de la surface transparente, la puissance totale $P$ du laser peut s'écrire :

soit :

$$
P \simeq A_{1}^{2} 2 S
$$

$$
A_{1}^{2} \simeq P / 2 S \text {. }
$$

La dimension du diaphragme limitant la partie du maximum de corrélation accessible au détecteur doit être proportionnelle à une fraction du diamètre $d$ des éléments les plus petits de la fonction $f\left(x_{1}, y_{1}\right)$. Le signal $E$ fourni par le détecteur est donc proportionnel à $d^{2}$ et a pour expression :

$$
E=k\left(\frac{\Delta t_{2}}{4}\right)^{2} \frac{P}{2 S} d^{2}
$$

ou encore :

$$
E=k\left(\frac{\Delta t_{2}}{4}\right)^{2} \frac{P}{2} \frac{1}{N}
$$

où $N$ est le nombre d'éléments indépendants de surface $d^{2}$.

Il apparaît donc que le signal recueilli par le détecteur au maximum de corrélation a une amplitude inversement proportionnelle au nombre d'éléments contenus par un signal transparent ou opaque.

Le même calcul en holographie ordinaire aboutit au même résultat avec le dynamique $\Delta t_{1}$ d'une courbe de réponse similaire à celle de la figure 4 .

Cas particulier de signaux aléatoires à composante continue nulle, analogues aux codes pseudo-aléatoires utilisés en radar. - En radar, on cherche à utiliser des signaux à grand gain de corrélation. Ce gain étant précisément égal au nombre d'éléments indépendants que comporte le signal à corréler, la formule (16) semble de mauvais augure. En fait, un signal pseudoaléatoire comme celui de la figure 12 a, dont la transparence des digits élémentaires est 1 ou 0 , peut être décomposé en un code à composante continue nulle, présentant les transparences $+1 / 2$ et $-1 / 2$ et en un créneau de longueur égale à celle du code et de transparence $+1 / 2$. La transformée de Fourier d'un tel code $(N=63$ digits) comporte donc un maximum central très intense correspondant au créneau $+1 / 2$, dont l'amplitude maximum est proportionnelle à 63 et un terme bien plus large correspondant au code $(+1 / 2,-1 / 2)$ dont l'amplitude maximum est proportionnelle à $\sqrt{63}$.
La contribution du créneau d'amplitude $1 / 2$ au maximum de corrélation est égale à la contribution du code $(+1 / 2,-1 / 2)$. Si, dans ces conditions, on enregistre le filtre adapté de façon que la dynamique linéaire du processus photographique corresponde à l'excursion du maximum de la transformée de Fourier du code $(+1 / 2,-1 / 2)$, tandis que la partie correspondant à la transformée de Fourier du créneau est arrêtée par un stop, la fonction d'autocorrélation obtenue ne comporte que le terme provenant du code $(+1 / 2,-1 / 2)$; mais son amplitude est multipliée par un facteur $\sqrt{63}$, car la dynamique utile dévolue à la transformée de Fourier du code $(+1 / 2$, $-1 / 2)$ est alors $\Delta t_{2}$ au lieu de $\Delta t_{2} / \sqrt{63}$.

Par rapport au maximum de la fonction d'autocorrélation du code $(1,0)$ qui obéit à la forme (15), l'amplitude du maximum est donc multipliée par un facteur $\sqrt{63} / 2$ et son intensité par un facteur $63 / 4$, soit $N / 4$.

La formule (16), qui est aussi valable pour des signaux à une dimension, devient donc, pour des fonctions du type $(+1 / 2,-1 / 2)$ à composante nulle comportant $N$ éléments :

$$
E=k\left(\frac{\Delta t_{2}}{4}\right)^{2} \frac{P}{2} \frac{1}{N} \frac{N}{4}
$$

d'où :

$$
E=\frac{k}{128}\left(\Delta t_{2}\right)^{2} P
$$

Cette formule (17) exprime donc que la fraction de l'énergie de l'ensemble du faisceau laser, qui peut être recueillie sur un maximum de corrélation par un détecteur, est constante quel que soit le nombre d'éléments indépendants du signal à corréler si le niveau de ces éléments, susceptible de prendre deux valeurs, est choisi de façon aléatoire ou pseudo-aléatoire et si la valeur moyenne du signal est nulle. Cette fraction est sensiblement égale à $1 / 2000$ pour des valeurs respectives de $k$ et $\Delta t_{2}$ de l'ordre de $1 / 10$ et 0,7 .

VÉRIFIGATION EXPÉRIMENTALE. - La formule (15) a été vérifiée pour l'énergie totale contenue dans la fonction d'autocorrélation d'un rectangle de largeur $a$ et de longueur $b$.

Dans ce cas, la formule (15) donne :

$$
\Phi_{f f}\left(x_{3}, y_{3}\right)=\Phi_{f f}(0,0) \operatorname{Tr} \frac{x_{3}}{a} \operatorname{Tr} \frac{y_{3}}{b}
$$

soit en intensité :

$$
\left[\Phi_{f f}\left(x_{3}, y_{3}\right)\right]^{2}=\left[\Phi_{f f}(0,0) \operatorname{Tr} \frac{x_{3}}{a} \operatorname{Tr} \frac{y_{3}}{b}\right]^{2}
$$

où $\operatorname{Tr}\left(x_{3} / a\right)$ et $\operatorname{Tr}\left(y_{3} / b\right)$ indiquent des fonctions triangles de largeur à mi-hauteur respectivement égales à $a$ et à $b$. 
D'où la puissance totale contenue dans la fonction de corrélation :

$P_{\mathrm{c}}=4\left[\Phi_{f f}(0,0)\right]^{2}$

$$
\int_{0}^{a} \int_{0}^{b}\left(-\frac{x_{3}}{a}+1\right)\left(-\frac{y_{3}}{b}+1\right) \mathrm{d} x_{3} \mathrm{~d} y_{3}
$$

donc : $\quad P_{\mathrm{c}}=4\left(\frac{A_{1} \Delta t_{2}}{4}\right)^{2} \frac{a b}{9}$.

La puissance diaphragmée par le rectangle objet, $P_{0}$, vaut :

$$
P_{0}=A_{1}^{2} a b .
$$

D'où le rapport :

soit $P_{\mathrm{c}} / P_{0}=\left(\Delta t_{2} / 6\right)^{2}$.

$$
\frac{P_{\mathrm{c}}}{P_{0}}=\frac{\left(\frac{A_{1} \Delta t_{2}}{2}\right)^{2} \frac{a b}{9}}{A_{1}^{2} a b}
$$

La mesure expérimentale de ce rapport permet de déterminer facilement si la dynamique effectivement utilisée pour un certain filtre adapté est correcte.

Le rapport obtenu avec le meilleur filtre adapté à un signal rectangulaire de $0,4 \times 6 \mathrm{~mm}$ a été de $0,75 / 100$. Il lui correspond donc une dynamique :

$$
\begin{aligned}
\Delta t_{2} & =(6 / 100) \sqrt{75} . \\
& \simeq 0,55 .
\end{aligned}
$$

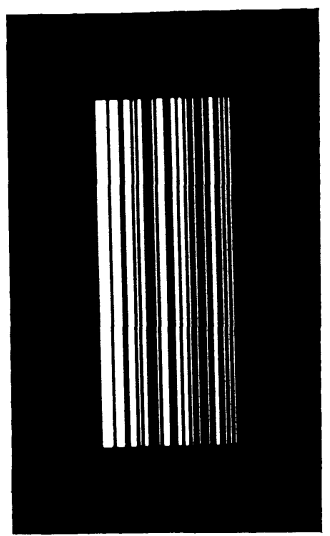

FIG. 12 a. - Code à 63 digits.

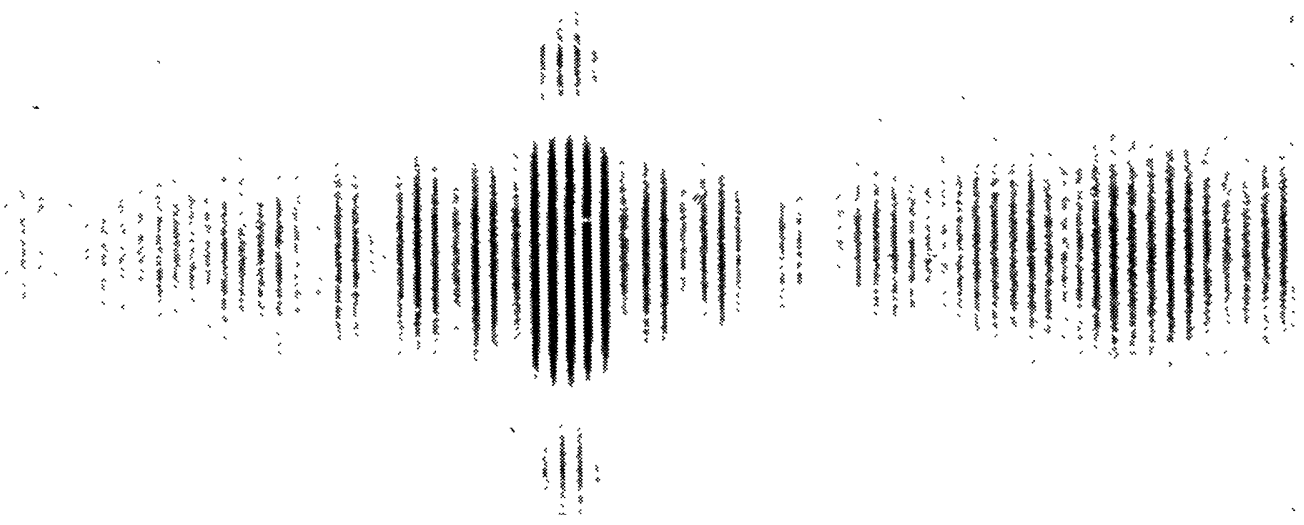

FIG. 12 b. - Filtre adapté au code à 63 digits $(1,0)$.

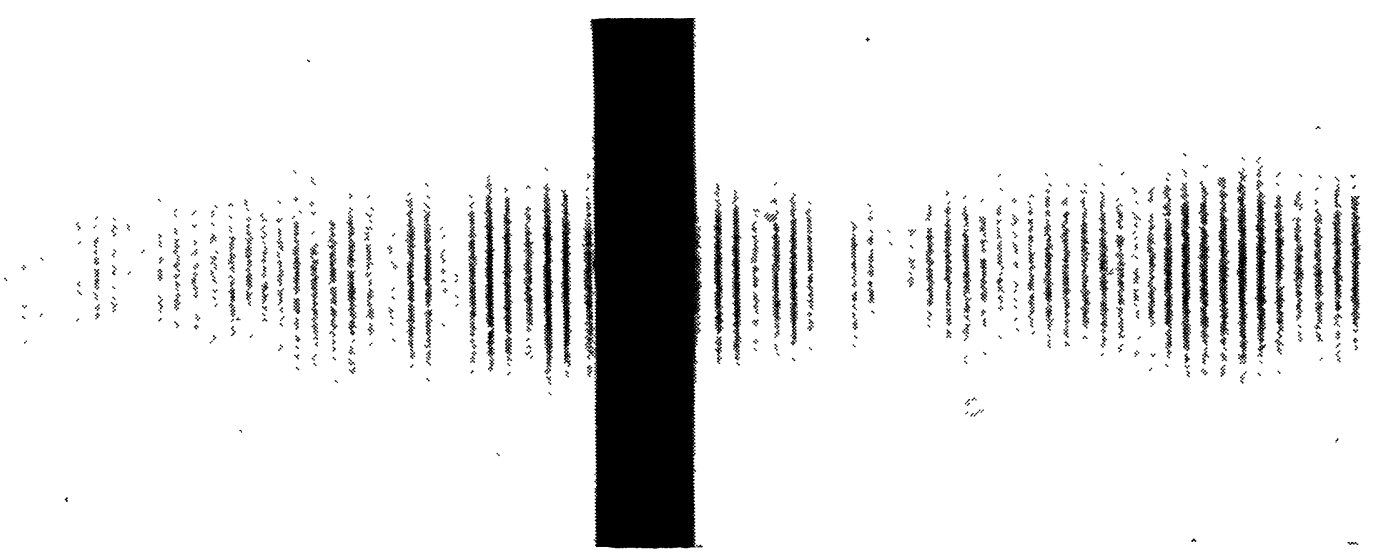

FIG. 12 c. - Filtre adapté au code à 63 digits $(+1 / 2,-1 / 2)$. 


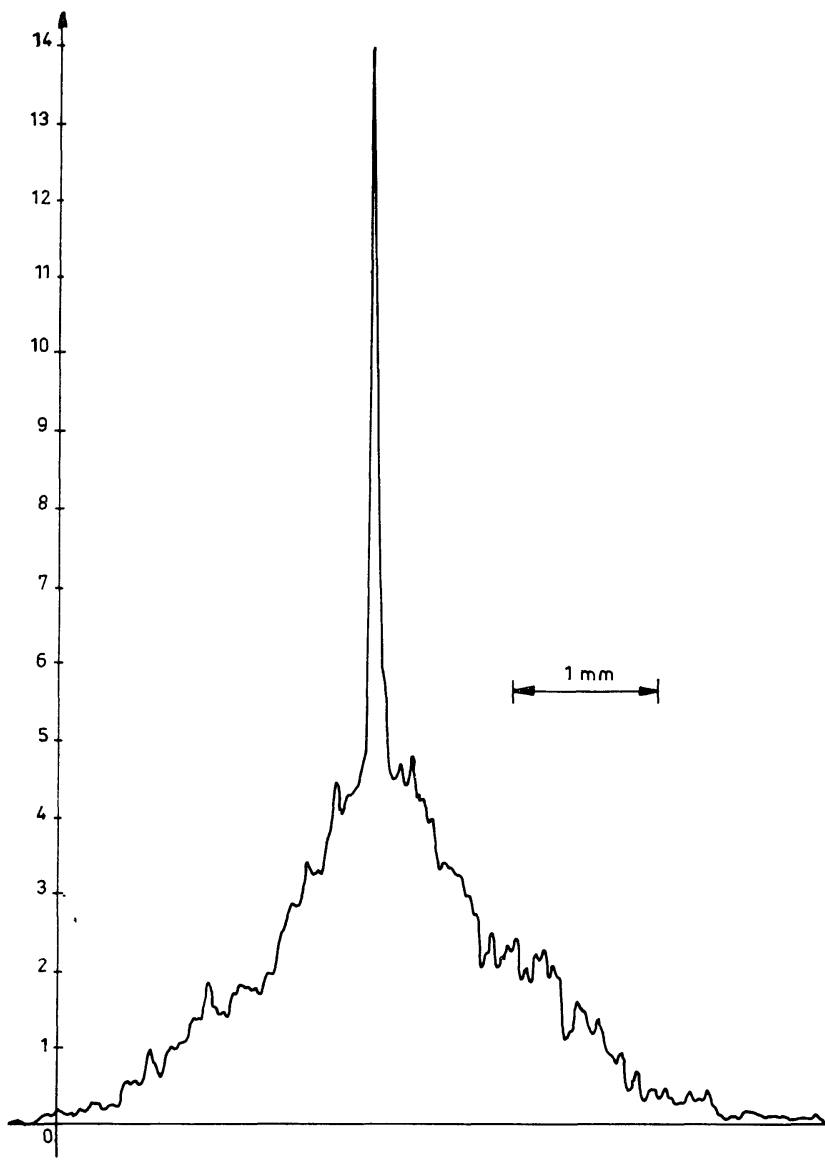

FIG. 13 a.

Fonction d'autocorrélation du code à 63 digits $(1,0)$.

Cette valeur assez satisfaisante pourrait certainement être portée à la dynamique de 0,7 du processus photographique en disposant d'un atténuateur d'ajustement de l'amplitude du maximum de la transformée de Fourier d'une valeur légèrement inférieure.

La fonction pseudo-aléatoire sur laquelle une augmentation de luminosité est mise en évidence en supprimant la composante continue est le code à 63 digits de la figure $12 \mathrm{a}$. Le filtre adapté au code $(1,0)$ est donné par la figure $12 \mathrm{~b}$ et le filtre adapté au code $(+1 / 2,-1 / 2)$, par la figure $12 \mathrm{c}$.

Les fonctions d'autocorrélation obtenues respectivement avec ces deux filtres sont données par les enregistrements de la figure 13.

Il apparaît un gain en luminosité de 6 contre un gain théorique de 16 , la différence étant due au fait que la dynamique réellement utilisée est inférieure de $40 \%$ à la dynamique exploitée pour le filtre adapté au code $(1,0)$.

Conclusion. - L'énergie totale contenue dans une image obtenue à partir d'un hologramme de Fourier est inversement proportionnelle au nombre d'éléments indépendants de cette image. En reconnaissance des

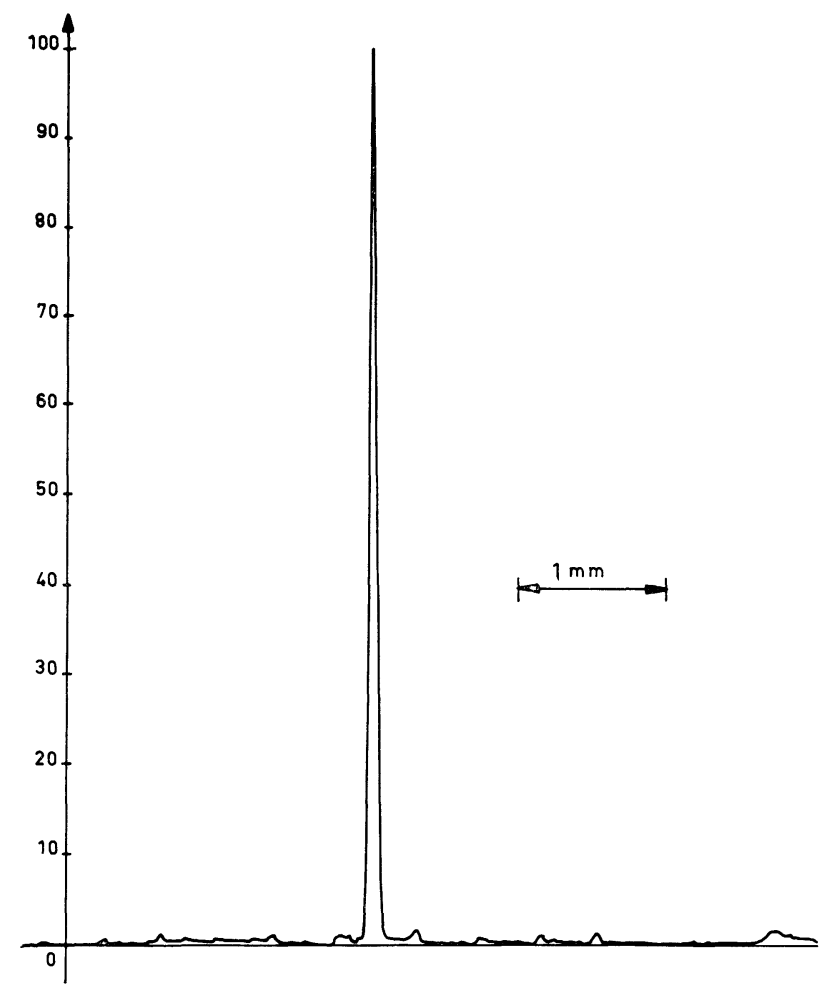

FIG. 13 b. - Fonction d'autocorrélation du code à 63 digits $(+1 / 2,-1 / 2)$.

formes, le même résultat est obtenu pour la puissance lumineuse disponible au maximum de corrélation quand les signaux utilisés sont positifs comme c'est le cas avec des images photographiques ordinaires. Par contre, en modifiant les fonctions employées de façon à les ramener à des signaux du type pseudo-aléatoire à valeur moyenne nulle, analogues aux codes pseudoaléatoires des radars modernes à corrélation, cette puissance reste constante alors que le gain de corrélation propre à l'objet initial est conservé.

Le procédé d'holographie employé est d'une mise en œuvre expérimentale délicate, mais il présente, du fait de son caractère linéaire, une supériorité potentielle certaine. En effet, la réponse impulsionnelle d'un hologramme réalisé par la méthode de l'affichage symétrique ne comporte pas de terme central indésirable et les termes parasites les plus importants provenant des non-linéarités du processus photographique ne se forment pas sur l'image utile. La luminosité des images restituées est donc supérieure, à performances égales par ailleurs.

Remerciements. - Nous remercions M. MichelH. Carpentier qui nous a suggéré la méthode de l'holographie symétrique, MM. Georges Pircher et Jean Dansac pour l'intérêt qu'ils ont porté à ce travail, M. Edgar Picquendar, directeur du Laboratoire de Recherches Générales de la Compagnie G.F.T.H.H.B. pour l'autorisation du publier ce travail, ainsi 
que M. Jean-Pierre Leborgne qui a effectué la majeure partie d'un travail expérimental particulièrement délicat.

Cette étude a été effectuée avec le soutien financier de la Direction des Recherches et des Moyens d'Essais.

\section{Annexe}

Termes parasites introduits par les non-linéarités du processus photographique en holographie de Fourier

Cas de l'holographie symétrique. - Le développement limité de (6) peut s'écrire :

$$
\begin{aligned}
t(U, V)=t_{0}+k_{1} F^{\prime \prime}(U, V) & +k_{2}\left[F^{\prime \prime}(U, V)\right]^{2} \\
& +k_{3}\left[F^{\prime \prime}(U, V)\right]^{3}+\ldots
\end{aligned}
$$

avec :

$$
\begin{aligned}
F^{\prime \prime}(U, V)=\frac{A_{1}^{\prime}}{\lambda f}\left[F(U, V) \mathrm{e}^{2 \pi j\left(U a_{2}+V b_{2}\right)}\right. & \\
& \left.+F^{*}(U, V) \mathrm{e}^{-2 \pi j\left(U a_{2}+V b_{2}\right)}\right] .
\end{aligned}
$$

Soit, la parenthèse ( ) remplaçant $\left(U a_{2}+V b_{2}\right)$ :

$$
\begin{aligned}
& t(U, V)=t_{0}+k_{1} \frac{A_{1}^{\prime}}{\lambda f}\left[F \mathrm{e}^{2 \pi j()}+F^{*} \mathrm{e}^{-2 \pi j()}\right] \\
&+ k_{2}\left(\frac{A_{1}^{\prime}}{\lambda f}\right)^{2}\left[F^{2} \mathrm{e}^{4 \pi_{j}()}+F^{* 2} \mathrm{e}^{-4 \pi_{j}()}+F F^{*}\right] \\
&+k_{3}\left(\frac{A_{1}^{\prime}}{\lambda f}\right)^{3}\left[F^{3} \mathrm{e}^{6 \pi_{j}()}+F^{* 3} \mathrm{e}^{-6 \pi_{j}()}+3 F^{2} F^{*} \mathrm{e}^{2 \pi j()}\right. \\
&\left.+3 F F^{* 2} \mathrm{e}^{-2 \pi_{j}()}\right] .
\end{aligned}
$$

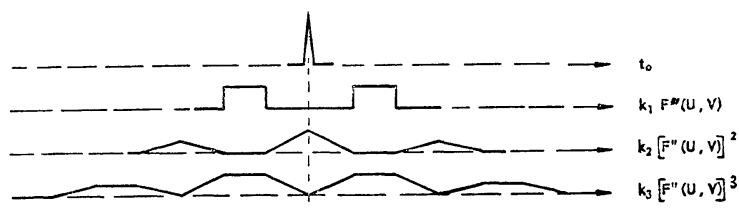

a) EN HOLOGRAPHIE SYMÉTRIQUE

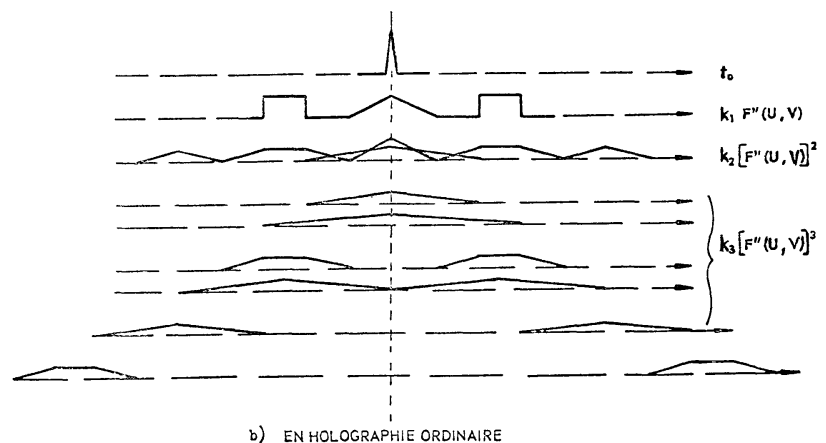

FIG. 14. - Termes fournis par un processus photographique non linéaire.
La réponse impulsionnelle d'un tel filtre contient donc les termes dont la position et l'encombrement sont donnés par la figure 14 a.

Cas de l'holographie ordinaire. - Le développement est identique avec :

$$
F^{\prime \prime}(U, V)=F^{\prime}(U, V) F^{\prime *}(U, V)-A_{\mathrm{p}}^{2}
$$

d'où :

$$
\begin{aligned}
F^{\prime \prime}(U, V)=\frac{A_{1}^{\prime}}{(\lambda f)}\left[\frac{A_{1}^{\prime}}{\lambda f} F(\right. & U, V) F^{*}(U, V) \\
& +A_{\mathrm{p}} F^{*}(U, V) \mathrm{e}^{-2 \pi_{j}\left(U a_{1}+V b_{1}\right)} \\
& \left.+A_{\mathrm{p}} F(U, V) \mathrm{e}^{+2 \pi_{j}\left(U a_{1}+V b_{1}\right)}\right]
\end{aligned}
$$

d'où, avec la parenthèse pour $\left(U a_{1}+V b_{1}\right)$ :

$t(U, V)$

$$
\begin{aligned}
& =t_{0}+k_{1} \frac{A_{1}^{\prime}}{\lambda f}\left[\frac{A_{1}^{\prime}}{\lambda f} F F^{*}+A_{\mathrm{p}} F^{*} \mathrm{e}^{-2 \pi_{j}()}+A_{\mathrm{p}} F \mathrm{e}^{2 \pi_{j}()}\right] \\
& +k_{2}\left[\frac{A_{1}^{\prime}}{\lambda f}\right]^{2}\left[\frac{A_{1}^{\prime 2}}{(\lambda f)^{2}} F^{2} F^{* 2}\right. \\
& +2 A_{\mathrm{p}}^{2} F F^{*}+2 \frac{A_{1}^{\prime}}{\lambda f} A_{\mathrm{p}} F F^{* 2} \mathrm{e}^{-2 \pi j()} \\
& +2 \frac{A_{1}^{\prime}}{\lambda f} A_{\mathrm{p}} F^{2} F^{*} \mathrm{e}^{+2 \pi_{j}()} \\
& \left.+A_{\mathrm{p}}^{2} F^{* 2} \mathrm{e}^{-4 \pi \jmath()}+A_{\mathrm{p}}^{2} F^{2} \mathrm{e}^{+4 \pi \jmath}()\right] \\
& +k_{3}\left[\frac{A_{1}^{\prime}}{\lambda f}\right]^{3}\left[\left(\frac{A_{1}^{\prime}}{\lambda f}\right)^{3} F^{3} F^{* 3}\right. \\
& +6 \frac{A_{1}^{\prime}}{\lambda f} A_{\mathrm{p}}^{2} F^{2} F^{* 2} \\
& +3\left(\left(\frac{A_{1}^{\prime}}{\lambda f}\right)^{2} A_{\mathrm{p}} F^{2} F^{* 3}+A_{\mathrm{p}}^{3} F F^{* 2}\right) \mathrm{e}^{-\cdot \pi} \\
& +3 \frac{A_{1}^{\prime}}{\lambda f} A_{\mathrm{p}}^{2} F F^{* 3} \mathrm{e}^{-4 \pi j()} \\
& +A_{\mathrm{p}}^{3} F^{* 3} \mathrm{e}^{-6 \pi j()} \\
& +3\left(\left(\frac{A_{1}^{\prime}}{\lambda f}\right)^{2} A_{\mathrm{p}} F^{3} F^{* 2}+A_{\mathrm{p}}^{3} F^{2} F^{*}\right) \mathrm{e}^{+2 \pi_{j}()} \\
& +3 \frac{A_{1}^{\prime}}{\lambda f} A_{\mathrm{p}}^{2} F^{3} F^{*} \mathrm{e}^{+4 \pi_{j}()} \\
& \left.+A_{\mathrm{p}}^{3} F^{3} \mathrm{e}^{+6 \pi_{j}()}\right] \text {. }
\end{aligned}
$$

La position et l'encombrement correspondant à ces différents termes sont donnés par la figure $14 \mathrm{~b}$. 


\section{BIBLIOGRAPHIE}

[1] Marḱchal (A.) et Françon (M.), Diffraction, structure des images, Revue d'Optique, Paris, 1960.

[2] Cutrona (L. J.), Lieith (E. N.), Palekmo (C. J.) et PORCELIO (L. J.), I.R.E. Transaction of Information Theory, juin 1960, p. 386.

[3] VAN DER LUGT (A.), I.E.E.E., Transaction of Information Theory, avril 1964, p. 139.

[4] CaRpentiter (M.-H.), L'onde électrique, 1966, 471, 740.
[5] VAN DER LUGT (A.), Optical and Electro-Optical Information Processing, The Massachusetts Institute of Technology Press.

[6] Leith (E. N.), Kozma (A.) et Upatnicks (J.), The laser letter, juin 1966, p. 2.

[7] VAN LigTEN (R. F.), J.O.S.A., 1966, 56, 1009.

[8] MARQUET (M.), Rapport D.R.M.E. 35165. 